02

\title{
Фосфоресценция жидкого кислорода при возбуждении на кооперативных переходах в видимой области спектра
}

\author{
() И.В. Багров ${ }^{1}$, Н.Г. Гоголева ${ }^{2}$, А.С. Гренишин ${ }^{1}$, В.М. Киселев ${ }^{1}$ \\ ${ }^{1}$ Государственный оптический институт им. С.И. Вавилова, \\ 199034 Санкт-Петербург, Россия \\ ${ }^{2}$ Санкт-Петербургский государственный электротехнический университет „ЛЭТИ “ им В.И. Ульянова (Ленина), \\ 197376 Санкт-Петербург, Россия \\ e-mail: kiselevvm21@gmail.com
}

Поступила в редакцию 18.09.2019 г.

В окончательной редакции 18.09.2019 г.

Принята к публикации 23.09.2019 г.

Проведено исследование фосфоресценции жидкого кислорода при его возбуждении на кооперативных переходах молекулярных комплексов кислорода $\mathrm{O}_{2}-\mathrm{O}_{2}$ с применением в качестве источников прямого оптического возбуждения светодиодных матриц с излучением в ближней УФ и видимой областях спектра с длинами волн, частично или полностью совпадающими с линиями поглощения молекулярных комплексов.

Ключевые слова: фосфоресценция, синглетный кислород, молекулярные комплексы, оптическое возбуждение, светодиодные матрицы.

DOI: $10.21883 /$ OS.2020.01.48838.267-19

\section{Введение}

Прямое оптическое возбуждение молекулярного кислорода может происходить как на одиночных молекулярных переходах, так и на кооперативных парных молекулярных переходах с участием комплексов $\mathrm{O}_{2}-\mathrm{O}_{2}$. Свойства этих переходов экспериментально исследованы в газовой фазе [1-4], в жидкой фазе [5-8], в твердой матрице [9-11] и в растворах [12-17] с помощью различных световых источников для прямого возбуждения молекул кислорода.

Для изучения переноса энергии возбуждения в отдельной молекуле кислорода или в паре молекул кислорода селективность оптического метода накачки превосходит другие методы возбуждения. В частности, как показано в [6,7], достаточно сильное поглощение жидким кислородом $\left(k=0.7 \mathrm{~cm}^{-1}\right)$ [18] выходного излучения $\mathrm{Nd}$ : YAG-лазера с модуляцией добротности на $\lambda=1.06 \mu \mathrm{m}$ обеспечивает большие концентрации молекул синглетного кислорода в состоянии ${ }^{1} \Delta_{g}$.

Генерация синглетного состояния кислорода $\left({ }^{1} \mathrm{O}_{2}\right)$ представляет особый интерес во множестве приложений, таких как тонкий химический синтез, катализ и очистка сточных вод, фотодинамическая терапия рака [19]. Для всех этих приложений остается актуальный вопрос об источниках оптического возбуждения молекулярного кислорода. Широкое применение получили лазерные источники излучения. Наряду с лазерными источниками нашли применение и светодиодные источники света. Как отмечается в работе [20], в световой терапии лазерные и светодиодные источники излучения оказались сопоставимыми при применении одинаковых доз светового облучения. При этом светодиоды имеют определенные преимущества перед лазерными источниками, заключающимися в простоте исполнения и в гораздо более низкой стоимости.

В данной работе показано, что с применением светодиодных источников в видимой области спектра можно получить эффективную генерацию синглетного кислорода при прямом возбуждении жидкого кислорода на кооперативных переходах молекулярных комплексов кислорода $\mathrm{O}_{2}-\mathrm{O}_{2}[21]$.

\section{Материалы и методика эксперимента}

При проведении исследований жидкий кислород находился в кварцевой кювете, охлаждаемой жидким азотом. Внутренний диаметр кюветы $36 \mathrm{~mm}$, длина $50 \mathrm{~mm}$.

В качестве источников оптического возбуждения жидкого кислорода использовались светодиодные матрицы $(26 \times 26 \mathrm{~mm})$ серии HPR40Е с длинами волн излучения, частично или полностью совпадающими с линиями поглощения молекулярных комплексов кислорода $\mathrm{O}_{2}-\mathrm{O}_{2}$ в видимой и ближней УФ областях спектра. Подводимая электрическая мощность для всех светодиодных матриц одинакова и равна $100 \mathrm{~W}$. Параметры излучения светодиодных матриц представлены в таблице. Для матриц с $\lambda_{m}=370,405,465,525$ и $625 \mathrm{~nm} \Delta \lambda_{0.5}=20 \mathrm{~nm}$, а для матрицы с $\lambda_{m}=600 \mathrm{~nm} \Delta \lambda_{0.5}=70 \mathrm{~nm}$ (рис. 1). Матрица с $\lambda_{m}=600 \mathrm{~nm}$ была использована в эксперименте для возбуждения кооперативного перехода на $\lambda_{m}=578 \mathrm{~nm}$. Как видно из рис. 2, эта матрица способна возбуждать сразу два кооперативных перехода на 578 и $633 \mathrm{~nm}$.

Кооперативные или димольные переходы комплекса $\mathrm{O}_{2}-\mathrm{O}_{2}$ для видимой и ближней УФ областей спектра приведены на рис. 2, который построен с ис- 
Параметры излучения светодиодных матриц

\begin{tabular}{c|c|c|c|c|c|c}
\hline$\lambda_{m}, \mathrm{~nm}$ & 370 & 405 & 480 & 525 & 600 & 625 \\
\hline$P, \mathrm{~W} / \mathrm{cm}^{2}$ & 0.35 & 1.0 & 1.8 & 0.60 & 1.0 & 1.0
\end{tabular}

пользованием материалов работ $[10,21,22]$. Соотношение максимумов в спектре поглощения кислорода на мономольных и димольных переходах в газовой фазе при давлении $150 \mathrm{~atm}$ и в твердой фазе приведено в работах $[13,23-25]$ в видимом, ближнем УФ и ближнем ИК диапазонах.

Фосфоресценция синглетного кислорода при оптическом возбуждении жидкого кислорода регистрировалась с использованием спектрометра М266.

\section{Результаты эксперимента и их обсуждение}

Основное внимание при регистрации фосфоресценция синглетного кислорода было сосредоточено на длине волны $1270 \mathrm{~nm}$, которая наиболее наглядно иллюстрирует эффективность генерации синглетного кислорода. Хотя другие линии также наблюдались, в частности на $\lambda=762$ и $1580 \mathrm{~nm}$. Наиболее интенсивная фосфоресценция синглетного кислорода в районе $\lambda=1270 \mathrm{~nm}$ наблюдалась при возбуждении жидкого кислорода на длинах волн 625 и $480 \mathrm{~nm}$. Соответствующие спектральные профили приведены на рис. 3, $a$. Следует заметить, что точное положение максимума фосфоресценции на рис. $3, a$ соответствует $1271.7 \mathrm{~nm}$, что, по-видимому, связано с наблюдаемым излучением молекулярного комплекса $\mathrm{O}_{2}-\mathrm{O}_{2}$. Аналогичный батохромный сдвиг наблюдается и для фосфоресценции в окрестности $762 \mathrm{~nm}$ при возбуждении жидкого кислорода на длине волны $480 \mathrm{~nm}$, как и в работе [26].

Соотношение между максимумами профилей на рис. 3, $a$ коррелирует с максимумами в спектре поглощения комплексов молекулярного кислорода (с учетом различия в плотности мощности излучения соответствующих светодиодных матриц), представленного на рис. 4. Спектр поглощения молекулярного кислорода высокого давления, приведенный на рис. 4, построен в соответствии с известными литературными данными [13,23-25]. Соотношение между максимумами, приведенными на рис. $3, a$, сохраняется и для максимумов фосфоресценции на длине волны $1586 \mathrm{~nm}$ (рис. 3, $b$ ). Это вполне естественно, так как спектральный переход, соответствующий данной длине волны, происходит с общего верхнего уровня для этих двух длин волн.

Корреляция между максимумами профилей фосфоресценции на длине волны $1270 \mathrm{~nm}$ с максимумами в спектре поглощения молекулярного кислорода сохраняется и для других длин волн оптического возбуждения с применением указанных выше светодиодных

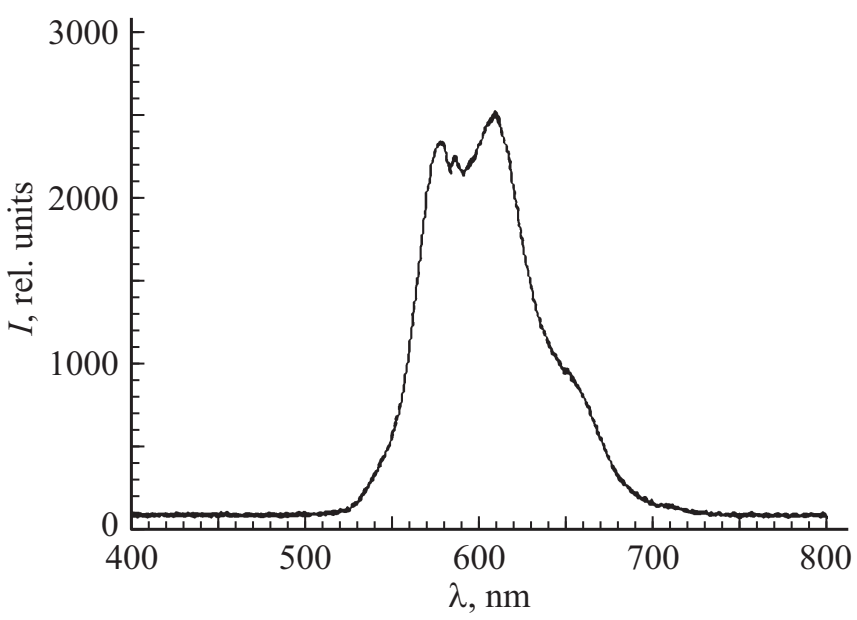

Рис. 1. Спектр излучения светодиодной матрицы c $\lambda_{m}=600 \mathrm{~nm}$.

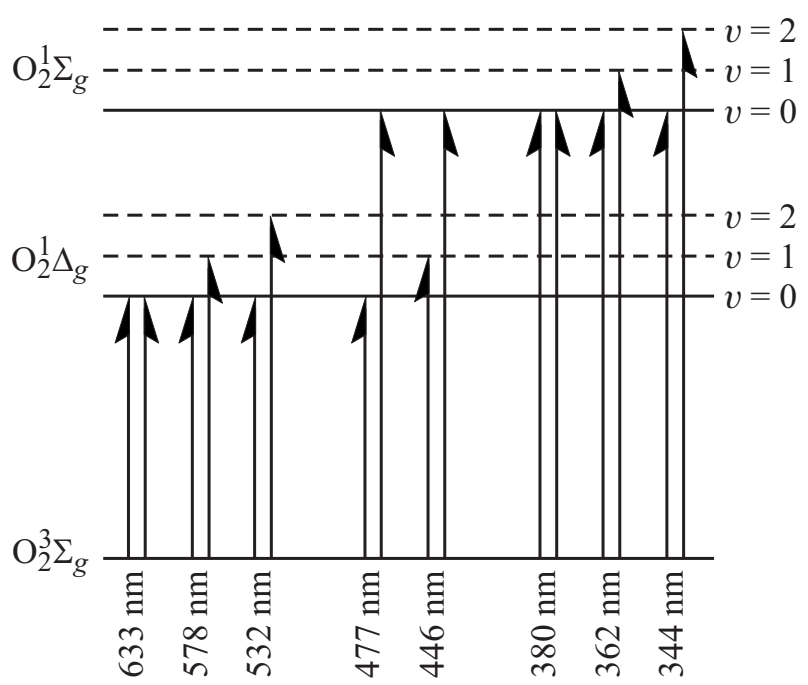

Рис. 2. Кооперативные переходы комплекса $\mathrm{O}_{2}-\mathrm{O}_{2}$.

матриц, длины волн излучения которых, как уже было отмечено выше, частично или полностью совпадают с полосами поглощения молекулярных комплексов кислорода $\mathrm{O}_{2}-\mathrm{O}_{2}$. Эту корреляцию можно видеть на рис. 5, на котором показаны значения интенсивностей в максимумах спектральных профилей фосфоресценции, отнесенные к плотности мощности светового потока на выходе конкретной светодиодной матрицы (кривая 1). Эти приведенные относительные интенсивности наглядно демонстрируют тесную корреляцию с зависимостью поглощения молекулярного кислорода от длины волны возбуждающего излучения (кривая 2), также построенной в относительном масштабе.

Из зависимости, приведенной на рис. 5, выпадает только значение эффективности фосфоресценции при возбуждении жидкого кислорода на длине волны $600 \mathrm{~nm}$ для светодиодной матрицы, спектр излучения которой не 
совсем корректно совпадает с максимумами поглощения молекулярного кислорода.

Интересно отметить, что характер этой спектральной зависимости для прямого возбуждения молекулярного кислорода в жидкой фазе не совсем корректно совпадает, а точнее совсем не совпадает с аналогичными зависимостями для прямого возбуждения молекулярного кислорода в различных органических растворителях [17] и молекулярного кислорода, сорбированного на поверхности твердофазного адсорбента [27], которые, кстати, друг с другом очень хорошо коррелируют. Причина этого различия, по-видимому, связана с тем, что в случае жидкого кислорода взаимодействие излучения фотовозбуждения происходит в основном только с комплексами $\mathrm{O}_{2}-\mathrm{O}_{2}$, в связи с чем наблюдается тесная корреляция интенсивности фосфоресценции с профилем поглощения молекулярного кислорода на димольных переходах, представленного на рис. 4. В случае же прямого возбуждения кислорода в органических растворителях и в сорбированном состоянии на поверхности адсорбента на интенсивность фосфоресценции синглетного кислорода преимущественное влияние оказывает спин-орбитальное взаимодействие молекулы кислорода
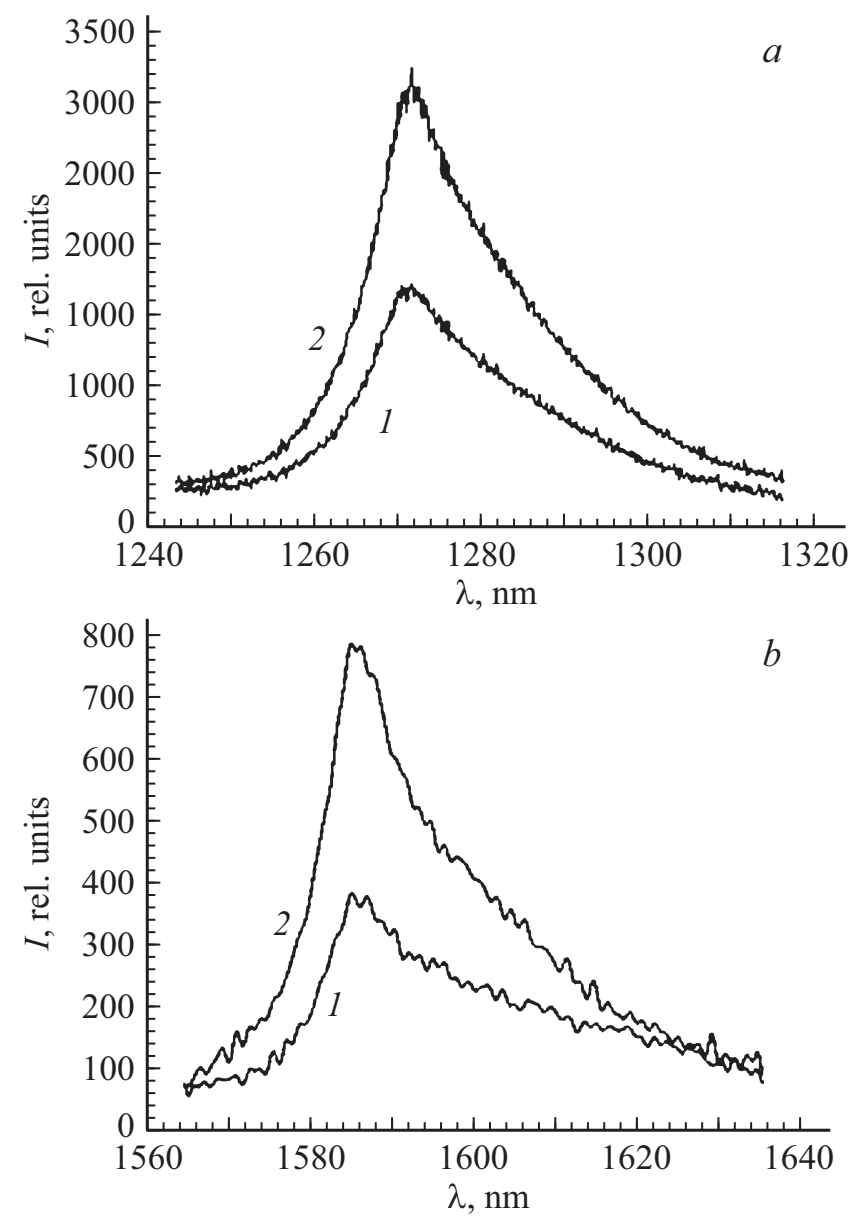

Рис. 3. Фосфоресценция жидкого кислорода на $\lambda=1270 \mathrm{~nm} \quad(a) \quad$ и $1586 \mathrm{~nm} \quad(b)$ при возбуждении на $\lambda_{m}=480 \mathrm{~nm}(1)$ и $625 \mathrm{~nm}(2)$.

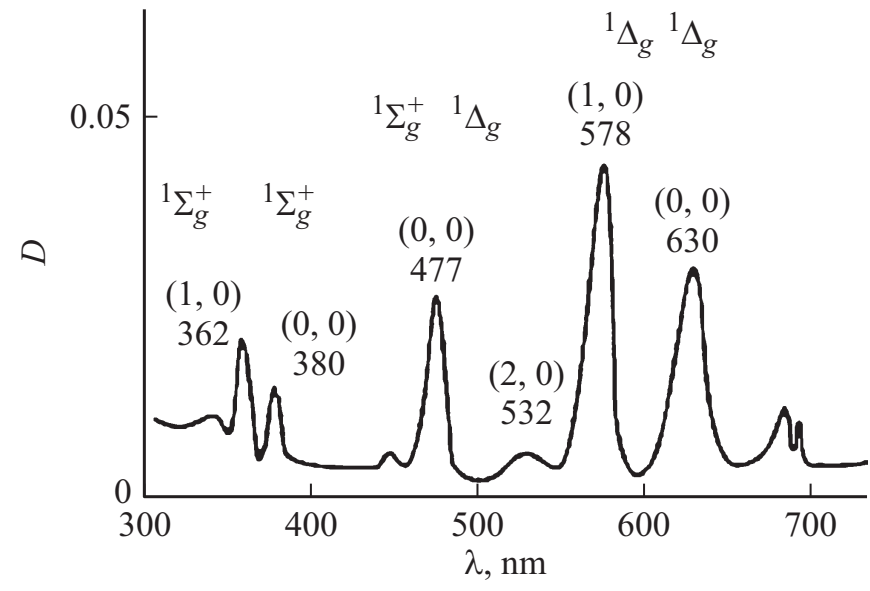

Рис. 4. Спектр поглощения молекулярного кислорода высокого давления на димольных переходах в ближнем УФ и видимом диапазонах.

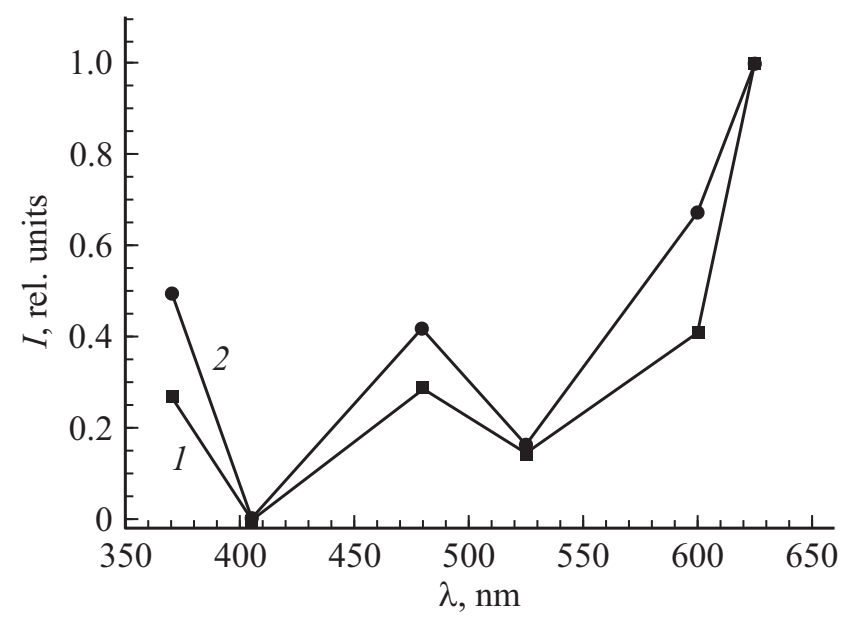

Рис. 5. Спектральная зависимость фосфоресценции синглетного кислорода при возбуждении жидкого кислорода светодиодным излучением (1) и спектральная зависимость поглощения молекулярного кислорода на кооперативных переходах (2).

с ее внешним окружением (с молекулами растворителя или адсорбента) [28-34]. Следствием этого, как показано в работах [28-34] и ряде других, является увеличение эффективности поглощения излучения оптической накачки комплексами кислорода с внешним окружением, а также повышение излучательной вероятности переходов из синглетных состояний молекулы кислорода в основное под воздействием внешнего окружения. Образование этих комплексов влияет и на формирование спектрального профиля поглощения, индуцированного столкновениями с внешним окружением [34].

Следует отметить примерно одинаковые наблюдаемые интенсивности фосфоресценции жидкого кислорода в данной работе и кислорода, растворенного в органическом растворителе в работах $[13,14]$, при значительно меньшей концентрации кислорода в органическом рас- 
творителе относительно жидкого состояния кислорода. По-видимому, это связано с более высоким временем жизни синглетного кислорода в растворителях и с активным тушением в жидком кислороде возбужденных состояний кислорода молекулами кислорода в основном состоянии и в процессе реакции пулинга при столкновении друг с другом двух возбужденных молекул кислорода или комплексов $\mathrm{O}_{2}-\mathrm{O}_{2}$ [5,35]. В работе [5] отмечается, что тушение комплексов было вдвое быстрее, чем одиночных возбужденных молекул кислорода.

\section{Заключение}

В результате выполненного исследования фосфоресценции жидкого кислорода при его возбуждении на кооперативных переходах молекулярных комплексов кислорода $\mathrm{O}_{2}-\mathrm{O}_{2}$ с применением в качестве источников прямого оптического возбуждения светодиодных матриц с излучением в ближней УФ и видимой областях спектра с длинами волн, частично или полностью совпадающими с линиями поглощения молекулярных комплексов, показано, что наблюдаемая спектральная зависимость фосфоресценции синглетного кислорода тесно коррелирует со спектральными профилями поглощения комплексов $\mathrm{O}_{2}-\mathrm{O}_{2}$. Отмечается существенное отличие этой спектральной зависимости от аналогичной спектральной зависимости фосфоресценции синглетного кислорода при прямом оптическом возбуждении кислорода в органических растворителях и в сорбированном состоянии на поверхности адсорбента.

Наблюдаемые батохромные сдвиги максимумов фосфоресценции синглетного кислорода относительно положения мономолекулярных переходов кислорода подтверждают, что они связаны в основном с излучением молекулярных комплексов $\mathrm{O}_{2}-\mathrm{O}_{2}$.

Приведено сравнение эффективности наблюдаемой фосфоресценции жидкого кислорода при его возбуждении светодиодными матрицами с фосфоресценцией синглетного кислорода в органических растворителях при аналогичных условиях оптического возбуждения.

\section{Конфликт интересов}

Авторы заявляют, что у них нет конфликта интересов.

\section{Список литературы}

[1] Matheson I.B.C., Lee J., Yamanashi B.S., Wolbarsht M.L. // Chem. Phys. Lett. 1974. V. 27. N 3. P. 355.

[2] Eisenberg W.C., Snelson A., Butler R., Taylor K., Murray R.U. // J. Photochemistry. 1984. V. 25. N 2-4. P. 439.

[3] Furui E., Akai N., Ida A., Kawai A., Shibuya K. // Chem. Phys. Lett. 2009. V. 471. P. 45.

[4] Trushina A.P., Goldort V.G., Kochubei S.A., Baklanov A.V. // Chem. Phys. Lett. 2010. V. 485. P. 11.
[5] Huestis D.L., Black G., Edelstein S.A., Sharpless R.L. // J. Chem. Phys. 1974. V. 60. P. 4471.

[6] Protz R., Maier M. // J. Chem. Phys. 1980. V. 73. N 11. P. 5464.

[7] Yamagishi A., Ohta T., Konno J., Inaba H. // J. Opt. Soc. Am. 1981. V. 71. N 10. P. 1197.

[8] Zhe S., Hui L., Canhua Z., Jinbo L., Xianglong C., Shu H., Baodong G., Dongjian Z., Dong L., Jingwei G., Yuqi J. // Proc. SPIE. 2015. V. 9255. P. 925529.

[9] Дианов-Клоков В.И. // Опт. и спектр. 1966. Т. 20. N 6. C. 954.

[10] Cooper P.D., Johnson R.E., Quickenden T.I. // Planetary and Space Science. 2003. V. 51. P. 183.

[11] Jockusch S., Turro N.J., Thompson E.K. etal. // Photochem. Photobiol. Sci. 2008. V. 7. P. 235.

[12] Krasnovsky A.A., Jr., Drozdova N.N., Ivanov A.V., Ambartzumian R.V. // Biochemistry (Moscow). 2003. V. 68. N 9. P. 963.

[13] Багров И.В., Белоусова И.М., Киселев В.М., Кисляков И.М., Соснов Е.Н. // Опт. и спектр. 2012. Т. 113. № 1. C. 594; Bagrov I.V., Belousova I.M., Kiselev V.M., Kislyakov I.M., Sosnov E.N. // Opt. Spectrosc. 2012. V. 113. N 1. P. 57-62.

[14] Багров И.В., Киселев В.М., Кисляков И.М., Соснов Е.Н. // Опт. и спектр. 2014. Т. 116. № 4. С. 609; Bagrov I.V., Kiselev V.M., Kislyakov I.M., Sosnov E.N.. Opt. Spectrosc. 2014. V. 116. N 4. P. 567-574.

[15] Krasnovsky A.A., Jr., Kozlov A.S. // Biophysics (Moscow). 2014. V. 59. N 2. P. 199.

[16] Bregnhфj M., Blázquez-Castro A., Westberg M., Breitenbach T., Ogilby P.R. // J. Phys. Chem. B. 2015. V. 119. N 17. P. 5422.

[17] Киселев В.М., Кисляков И.М., Багров И.В. // Опт. и спектр. 2016. Т. 120. № 6. С. 916; Kiselev V.M., Kislyakov I.M., Bagrov I.V. // Opt. Spectrosc. 2016. V. 120. N 6. P. 859.

[18] Dianov-Klokov V.I. // Opt. Spectrosc. 1959. V. 6. P. 290.

[19] Pibiri I., Buscemi S., Piccionello A.P., Pace A. // Chem. Photochem. 2018. V. 2. N 7. P. 535.

[20] Spitler R., Berns M.W. // J. Biomedical Optics. 2014. V. 19(3). P. 038001.

[21] Разумовский С.Д. Кислород - элементарные формы и свойства. М.: Химия, 1979.

[22] Blázquez-Castro A. // Redox Biology. 2017. V. 13. P. 39.

[23] Гуринович Г.П. // Журн. прикл. спектр. 1991. Т. 54. № 3. C. 403 .

[24] Greenblatt G.D., Orlando J.J., Burkholder J.B., Ravishankara A.R. // J. Geophysical Res. 1990. V. 95. ND11. P. 18577.

[25] Jockusch S., Turro N.J., Thompson E.K., Gouterman M., Callis J.B., et al. // Photochem. Photobiol. Sci. 2008. V. 7. P. 235.

[26] Novick S.E., Broida H.P. // J. Chem. Phys. 1977. V. 67. N 12. P. 5975.

[27] Киселев В.М., Кисляков И.М., Бурчинов А.Н. // Опт. и спектр. 2016. Т. 120. № 4. С. 545; Kiselev V.M., Kislyakov I.M., Burchinov A.N. // Opt. Spectrosc. 2016. V. 120. N 4. P. 520.

[28] Минаев Б.Ф. // Изв. вузов. Сер. Физ. 1978. № 9. С. 115.

[29] Минаев Б.Ф. // Опт. и спектр. 1985. Т. 58. В. 6. С. 1238.

[30] Scurlock R.D., Ogilby P.R. // J. Phys. Chem. 1987. V. 91. P. 4599.

[31] Schmidt R., Afshari E. // J. Phys. Chem. 1990. V. 94. P. 4377. 
[32] Minaev B.F., Lunell S., Kobzev G. // J. Mol. Struct. Theochem. 1993. V. 284. P. 1.

[33] Минаев Б.Ф. // Успехи химии. 2007. Т. 76. N 11. С. 1059.

[34] Bregnhøj M., Westberg M., Minaev B.F., Ogilby P.R. // Acc. Chem. Res. 2017. V. 50. N 8. P. 1920.

[35] Kumar A., Rajesh R., Singhal G., Tyagi R.K. // Appl. Phys. B. 2007. V. 89. P. 385. 